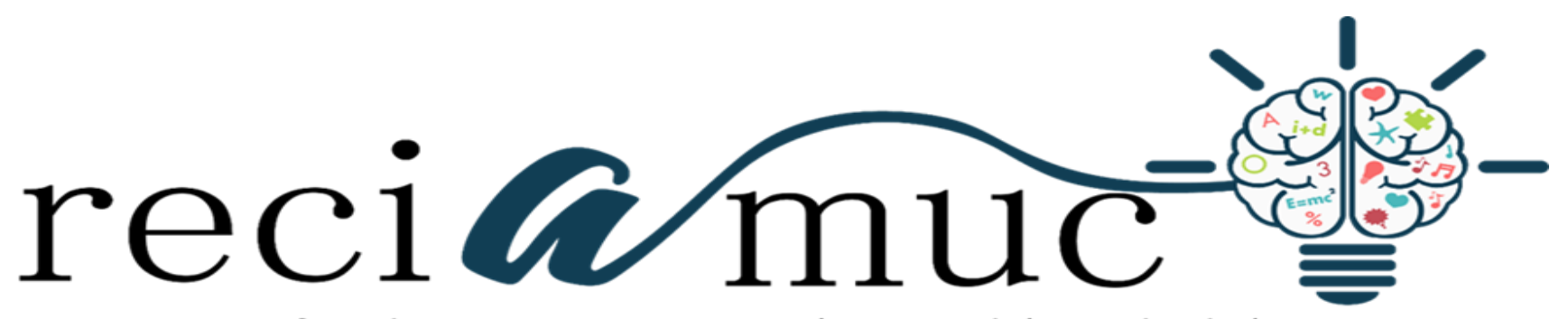

Revista científica de investigación actualización del mundo de las ciencias

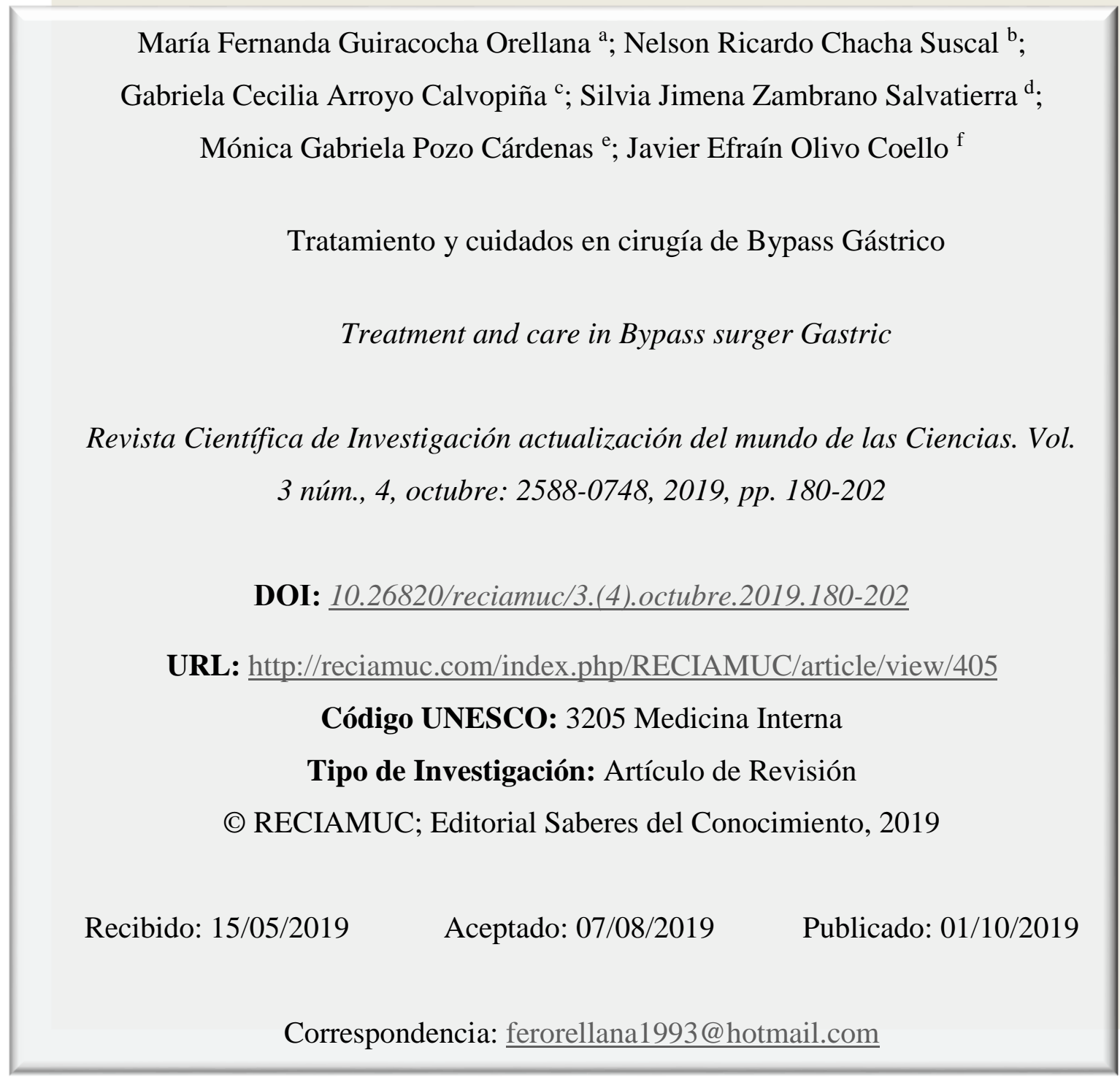

a. Médica; ferorellana1993@ @otmail.com; Cuenca - Ecuador.

b. Médico; Clínica CLIESMETROSA; ricardocsmed93@gmail.com; Cuenca - Ecuador.

c. Médica Cirujana; Médico general en funciones hospitalarias en el servicio de neonatología - Hospital general Marco Vinicio Iza; gaby_arca@hotmail.com; Lago Agrio - Ecuador.

d. Médica Cirujana; Médico residente del Hospital Básico San Andrés; silviajimena1993@ gmail.com; Flavio Alfaro - Ecuador.

e. Médico General; Médico residente del servicio de cirugía general - Hospital Marco Vinicio Iza; medicopozo@gmail.com; Lago Agrio - Ecuador.

f. Médico Cirujano; Médico residente en funciones hospitalarias área cirugía - Hospital Básico Baños; javy_o29@hotmail.com; Baños de Agua Santa - Ecuador 


\section{Tratamiento y cuidados en cirugía de Bypass Gástrico}

Vol. 3, núm. 4., (2019)

María Fernanda Guiracocha Orellana; Nelson Ricardo Chacha Suscal; Gabriela Cecilia Arroyo Calvopiña; Silvia Jimena Zambrano Salvatierra; Mónica Gabriela Pozo Cárdenas; Javier Efraín

Olivo Coello

\section{RESUMEN}

El término cirugía bariátrica define al conjunto de intervenciones quirúrgicas diseñadas para producir pérdidas importantes de peso, como lo es el bypass gástrico, entendido como un proceso quirúrgico que permite al paciente adelgazar modificando la forma en la que el estómago y el intestino delgado manejan el alimento consumido. A través del bypass gástrico, se reduce el tamaño del estómago de tal forma que el paciente siente saciedad con menor cantidad de comida. La intervención, que puede realizarse bien con cirugía abierta, o con cirugía laparoscopia, se lleva a cabo en dos pasos. En la cirugía, el cirujano procederá a dividir el estómago en dos partes, una superior, más pequeña, y otra inferior mayor. La sección pequeña se denomina bolsa y es donde se digerirán los alimentos que se van a consumir. Seguidamente, se da la segunda parte de la intervención, denominada derivación, en donde el cirujano conectará la bolsa con el intestino delgado saltando un fragmento, de manera que solo se empleará cerca del 60\%. De esta manera, además de reducir la cantidad de alimentos que se toman, se disminuye también la absorción de calorías en el intestino. La consecuencia directa de esta técnica es perder peso. Sus cuidados y tratamiento estarán centrados particularmente en el reposo y la dieta del paciente, la cual está diseñada para lo siguiente: Permitir que el estómago del paciente sane sin que la comida que consumes lo estire, lograr que el paciente se acostumbre a comer las cantidades más pequeñas de alimentos que su estómago más pequeño puede digerir de manera cómoda y segura, ayudar al paciente a perder peso y evitar aumentar de peso y evitar los efectos secundarios y las complicaciones de la cirugía. Este tipo de atención permitirá, el paciente progresar en consistencia a una dieta triturada o blanda.

Palabras Claves: Tratamiento; Cuidados; Bypass Gástrico; Estómago; Obesidad; Dieta. 


\section{Tratamiento y cuidados en cirugía de Bypass Gástrico}

Vol. 3, núm. 4., (2019)

María Fernanda Guiracocha Orellana; Nelson Ricardo Chacha Suscal; Gabriela Cecilia Arroyo

Calvopiña; Silvia Jimena Zambrano Salvatierra; Mónica Gabriela Pozo Cárdenas; Javier Efraín Olivo Coello

\section{ABSTRACT}

The term bariatric surgery defines the set of surgical interventions designed to produce significant weight losses, such as gastric bypass, understood as a surgical process that allows the patient to lose weight by modifying the way in which the stomach and small intestine handle the food consumed Through the gastric bypass, the size of the stomach is reduced in such a way that the patient feels full with less food. The intervention, which can be performed either with open surgery, or with laparoscopic surgery, is carried out in two steps. In surgery, the surgeon will proceed to divide the stomach into two parts, an upper one, a smaller one, and a lower one. The small section is called the bag and is where the food to be consumed will be digested. Next, there is the second part of the intervention, called a shunt, where the surgeon will connect the bag with the small intestine by skipping a fragment, so that only about $60 \%$ will be used. In this way, in addition to reducing the amount of food that is taken, the absorption of calories in the intestine is also decreased. The direct consequence of this technique is to lose weight. Their care and treatment will be particularly focused on the patient's rest and diet, which is designed for the following: Allow the patient's stomach to heal without stretching the food you eat, get the patient to get used to eating the amounts Smaller food that your smaller stomach can digest comfortably and safely, help the patient lose weight and avoid gaining weight and avoid the side effects and complications of surgery. This type of care will allow the patient to progress in consistency to a crushed or soft diet.

Key Words: Treatment; Care; Gastric Bypass; Stomach; Obesity; Diet. 


\section{Tratamiento y cuidados en cirugía de Bypass Gástrico}

Vol. 3, núm. 4., (2019)

María Fernanda Guiracocha Orellana; Nelson Ricardo Chacha Suscal; Gabriela Cecilia Arroyo Calvopiña; Silvia Jimena Zambrano Salvatierra; Mónica Gabriela Pozo Cárdenas; Javier Efraín

Olivo Coello

\section{Introducción.}

La cirugía de la obesidad ha tenido un impresionante desarrollo en los últimos 20 años, debido a la necesidad de tratamiento efectivo y duradero para esta enfermedad crónica epidémica. La introducción de la técnica laparoscópica a las cirugías bariátricas contribuyó en forma importante a disminuir sus complicaciones y mortalidad. Las operaciones más frecuentes en el mundo son el bypass gástrico, la gastrectomía vertical, la banda gástrica ajustable y la derivación biliopancreática. (Vincent, 2015).

A partir de las recomendaciones del panel de consenso del National Institute of Health de los EE.UU, que datan de 1991, se produjo una rápida difusión del tratamiento quirúrgico de la obesidad. (Vincent, 2015). Sus ya conocidas recomendaciones de indicar el tratamiento quirúrgico para pacientes portadores de Índice de Masa Corporal (IMC) $>$ a $40 \mathrm{~kg} / \mathrm{m}^{2}$ y para pacientes que tienen un IMC > a $35 \mathrm{~kg} / \mathrm{m}^{2}$, pero con comorbilidades asociadas a la obesidad, han sido la referencia desde entonces.

Sin embargo, en los últimos años y debido a factores como: buenos resultados del tratamiento quirúrgico, disminución importante de la morbimortalidad, aplicación de la técnica laparoscópica con todos sus beneficios, pobres resultados de la terapia médica y difíciles de mantener al largo plazo; (Vianco, 2016) en la práctica clínica son cada vez más los centros de obesidad que indican el tratamiento quirúrgico para pacientes portadores de IMC entre 30 y $35 \mathrm{~kg} / \mathrm{m}^{2}$, especialmente sobre $32 \mathrm{~kg} / \mathrm{m}^{2}$, en la medida que tengan comorbilidades metabólicas y que constituyen parte importante del foco a tratar, como son la diabetes mellitus y dislipidemias severas.

En 1966 Mason describe el primer Bypass Gástrico desarrollado para producir baja de peso, distinto al conocido actualmente (consistía en una gastrectomía horizontal asociada a una gastroyeyunoanastomosis simple). (Herrsent, 2016). Posteriormente, en 1977, Alder y Terry correlacionaron el largo del remanente gástrico con la pérdida de peso. En el mismo año, Alden propuso solamente engrapar el estómago en forma horizontal, con el fin de disminuir la posibilidad de filtración, y Griffen introduce la configuración en Y de Roux para eliminar el reflujo biliar. En 


\section{Tratamiento y cuidados en cirugía de Bypass Gástrico}

Vol. 3, núm. 4., (2019)

María Fernanda Guiracocha Orellana; Nelson Ricardo Chacha Suscal; Gabriela Cecilia Arroyo

Calvopiña; Silvia Jimena Zambrano Salvatierra; Mónica Gabriela Pozo Cárdenas; Javier Efraín Olivo Coello

1994 Wittgrove y Clark describen los primeros pacientes operados de un bypass gástrico por vía laparoscópica.

Este método es definido y considerado mundialmente como el tratamiento quirúrgico estándar para la obesidad, por la gran cantidad de información que existe sobre esta operación y los buenos resultados reportados a muy largo plazo, en pacientes portadores de obesidad mórbida. Este tipo de cirugía consiste en la creación de un pequeño reservorio gástrico a expensas de la curvatura menor, de no más de $30 \mathrm{ml}$. de capacidad, asociado a una gastroyeyunoanastomosis a un asa defuncionalizada en Y de Roux.

\section{Imagen $\mathbf{N}^{\circ}$ 1. Técnica de Bypass Gástrico}

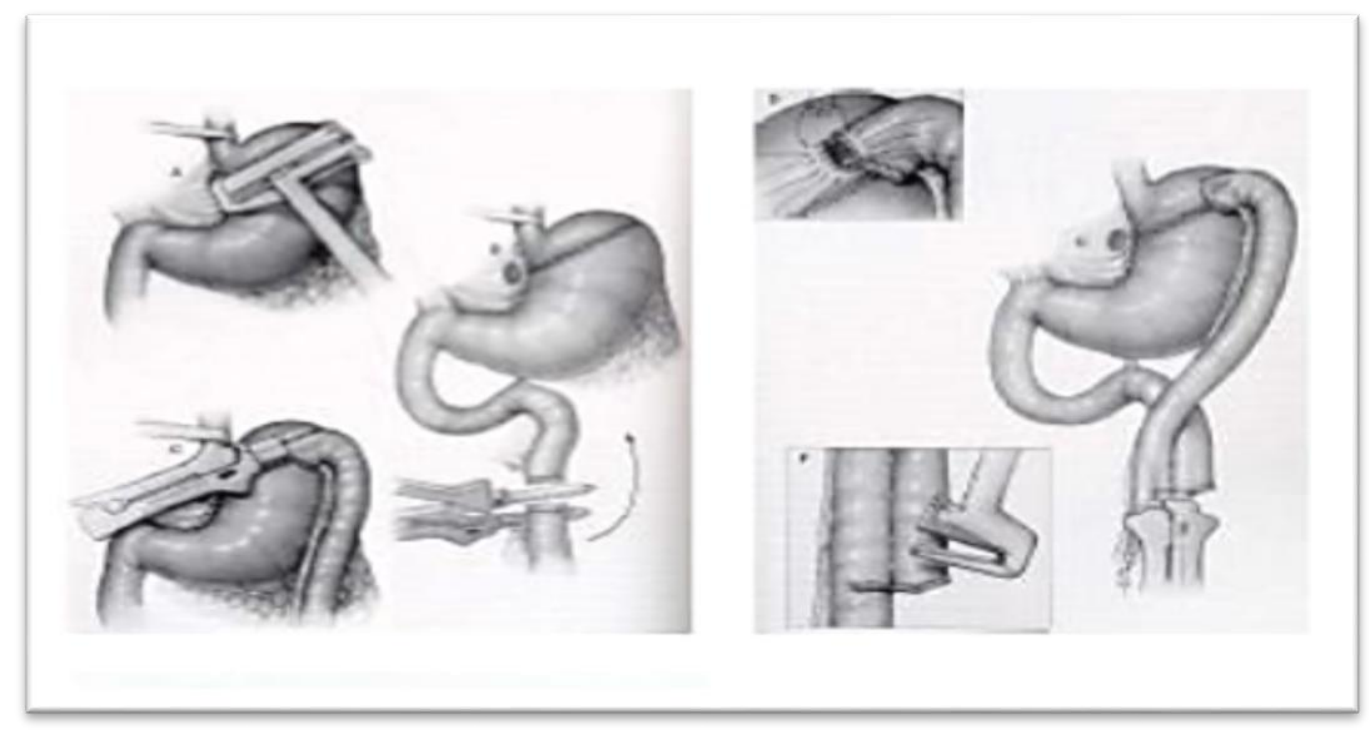

Fuente: (Cardozzo, 2017).

Según la Sociedad Española de Cirugía de la Obesidad Mórbida y de las Enfermedades Metabólicas (SECO), la cirugía de reducción de estómago está indicada para los pacientes con obesidad mórbida que presenten índices de masa corporal (IMC) superiores a 40kg/m2, o bien para aquellos con obesidad grave (IMC $>35 \mathrm{~kg} / \mathrm{m} 2)$ y que además tienen patologías asociadas. Con esta técnica se puede adelgazar entre 5-10 kg al mes en el primer año después de hacerse el bypass 


\section{Tratamiento y cuidados en cirugía de Bypass Gástrico}

Vol. 3, núm. 4., (2019)

María Fernanda Guiracocha Orellana; Nelson Ricardo Chacha Suscal; Gabriela Cecilia Arroyo Calvopiña; Silvia Jimena Zambrano Salvatierra; Mónica Gabriela Pozo Cárdenas; Javier Efraín Olivo Coello

gástrico. La pérdida de peso disminuirá con el tiempo y, como siempre, dependerá si el paciente reeduca sus hábitos dietéticos con una dieta y un plan de ejercicios adecuado. Según los expertos, se puede perder la mitad o más en los primeros dos años.

A través de esta cirugía, el estómago distal permanece in situ y queda excluido del tránsito alimentario. En este procedimiento han sido tres los aspectos técnicos de especial controversia: el tamaño de la bolsa gástrica, el diámetro de la anastomosis entre el estómago y el yeyuno, y el largo del asa defuncionalizada. En este tipo de casos, el tamaño de la bolsa gástrica resulta difícil de medir con certeza, pues si se mide en el periodo perioperatorio inmediato (la clásica radiografía contrastada del postoperatorio) está distorsionada por el proceso inflamatorio cicatrizal. (Cardozzo, 2017).

Por ende, el bypass gástrico es una técnica parcialmente malabsortiva que consiste en la creación de un pequeño reservorio gástrico de unos 15-30 ml. de capacidad al que se le anastomosa un asa de intestino delgado en Y de Roux. Esta asa de intestino se secciona a unos 60-70 cm. del ángulo de Treitz y se anastomosa al reservorio gástrico. La anastomosis del asa de la Y de Roux se realiza a una distancia que va desde $\operatorname{los} 75 \mathrm{~cm}$ a $\operatorname{los} 250 \mathrm{~cm}$. de la unión gastroyeyunal dependiendo del grado de obesidad del paciente. (Cardozzo, 2017).

En los últimos años, algunos grupos están realizando el bypass gástrico por vía laparoscópica con similares resultados en cuanto a la pérdida de peso. Las ventajas de realizar esta técnica por vía laparoscópica son una menor estancia hospitalaria, una rápida incorporación a la vida laboral y una disminución del re intervenciones posteriores secundarias a las eventraciones. De ahí, la importancia del presente artículo, el cual centra su atención en analizar cómo es su tratamiento y sus cuidados post operatorios

\section{Tipo de Investigación.}

Dentro de toda práctica investigativa, se precisan acciones de carácter metodológico mediante las cuales, se logra conocer y proyectar los eventos posibles que la determinan, así como las características que hacen del acto científico un proceso interactivo ajustado a una realidad posible 


\section{Tratamiento y cuidados en cirugía de Bypass Gástrico}

Vol. 3, núm. 4., (2019)

María Fernanda Guiracocha Orellana; Nelson Ricardo Chacha Suscal; Gabriela Cecilia Arroyo

Calvopiña; Silvia Jimena Zambrano Salvatierra; Mónica Gabriela Pozo Cárdenas; Javier Efraín Olivo Coello

de ser interpretada. En este sentido, se puede decir, que la presente investigación corresponde al tipo documental, definido por (Dávila, 2012), "se ocupa del estudio de problemas planteados a nivel teórico, la información requerida para abordarlos se encuentra básicamente en materiales impresos, audiovisuales y /o electrónicos”. (p.41).

En consideración a esta definición, la orientación metodológica permitió la oportunidad de cumplir con una serie de actividades inherentes a la revisión y lectura de diversos documentos donde se encontraron ideas explicitas relacionadas con los tópicos encargados de identificar a cada característica insertada en el estudio. Por lo tanto, se realizaron continuas interpretaciones con el claro propósito de revisar aquellas apreciaciones o investigaciones propuestas por diferentes investigadores, para luego dar la respectiva argumentación a los planteamientos, en función a las necesidades encontradas en la indagación.

\section{Fuentes Documentales.}

El análisis correspondiente a las características que predomina en el tema seleccionado, llevan a incluir diferentes fuentes documentales encargadas de darle el respectivo apoyo y en ese sentido cumplir con la valoración de los hechos a fin de generar nuevos criterios que sirven de referencia a otros procesos investigativos. Para (Arias, 2010), las fuentes documentales incorporadas en la investigación documental o bibliográfica, "representa la suma de materiales sistemáticos que son revisados en forma rigurosa y profunda para llegar a un análisis del fenómeno". (p.41). Por lo tanto, se procedió a cumplir con la realización de una lectura previa determinada por encontrar aquellos aspectos estrechamente vinculados con la "TRATAMIENTO Y CUIDADOS EN CIRUGÍA DE BYPASS GÁTRICO” para luego explicar mediante un desarrollo las respectivas apreciaciones generales de importancia.

\section{Técnicas para la Recolección de la Información.}

La conducción de la investigación para ser realizada en función a las particularidades que determinan a los estudios documentales, tiene como fin el desarrollo de un conjunto de acciones encargadas de llevar a la selección de técnicas estrechamente vinculadas con las características del 


\section{Tratamiento y cuidados en cirugía de Bypass Gástrico}

Vol. 3, núm. 4., (2019)

María Fernanda Guiracocha Orellana; Nelson Ricardo Chacha Suscal; Gabriela Cecilia Arroyo Calvopiña; Silvia Jimena Zambrano Salvatierra; Mónica Gabriela Pozo Cárdenas; Javier Efraín Olivo Coello

estudio. En tal sentido, (Arias Ob cit) refiere, que es "una técnica particular para aportar ayuda a los procedimientos de selección de las ideas primarias y secundarias”. (p. 71).

Por ello, se procedió a la utilización del subrayado, resúmenes, fichaje, como parte básica para la revisión y selección de los documentos que presentan el contenido teórico. Es decir, que mediante su aplicación de estas técnicas se pudo llegar a recoger informaciones en cuanto a la revisión bibliográfica de los diversos elementos encargados de orientar el proceso de investigación. Tal como lo expresa, (Arias $\mathrm{Ob}$ cit) "las técnicas documentales proporcionan las herramientas esenciales y determinantes para responder a los objetivos formulados y llegar a resultados efectivos" (p. 58). Es decir, para responder con eficiencia a las necesidades investigativas, se introdujeron como técnica de recolección el método inductivo, que hizo posible llevar a cabo una valoración de los hechos de forma particular para llegar a la explicación desde una visión general.

Asimismo, se emplearon las técnicas de análisis de información para la realización de la investigación que fue ejecutada bajo la dinámica de aplicar diversos elementos encargados de determinar el camino a recorrer por el estudio, según, (Arias, Ob cit) las técnicas de procesamiento de datos en los estudios documentales "son las encargadas de ofrecer al investigador la visión o pasos que debe cumplir durante su ejercicio, cada una de ellas debe estar en correspondencia con el nivel a emplear" (p. 123). Esto indica, que, para llevar a cabo el procesamiento de los datos obtenidos, es necesario establecer las técnicas que serán seleccionadas, destacándose en este caso, de manera particular: fichas de resumen, textual, registros descriptivos entre otros, los mismos se deben ajustar al nivel que ha sido seleccionado.

\section{Resultados.}

La cirugía de baipás gástrico es el tipo de procedimiento quirúrgico más frecuente para adelgazar. El baipás gástrico y otros tipos de disecciones para adelgazar, conocidos en conjunto como "cirugía bariátrica", son desarrollados con el fin de realizar cambios en el estómago y el aparato digestivo, los cuales limitan la cantidad de alimentos que puedes comer y de nutrientes que la persona absorbe, lo que provoca el adelgazamiento. (Vianco 2016). 


\section{Tratamiento y cuidados en cirugía de Bypass Gástrico}

Vol. 3, núm. 4., (2019)

María Fernanda Guiracocha Orellana; Nelson Ricardo Chacha Suscal; Gabriela Cecilia Arroyo

Calvopiña; Silvia Jimena Zambrano Salvatierra; Mónica Gabriela Pozo Cárdenas; Javier Efraín Olivo Coello

Aunque pueda parecer atractiva, la cirugía de baipás gástrico no es para cualquiera. Como todo procedimiento importante, tiene riesgos para la salud y efectos secundarios considerables. Además, el éxito a largo plazo de la cirugía de baipás gástrico depende de la capacidad de la persona para hacer cambios permanentes en su estilo de vida; a razón de ello, los pacientes que quieren ser considerados para someterse a una cirugía de baipás gástrico deben someterse a una evaluación rigurosa para determinar si este procedimiento es apto para la situación de cada una.

Dicho de esta forma, el baipás gástrico y el resto de las cirugías para bajar de peso son procedimientos muy importantes que cambian la vida del paciente. Si bien la cirugía para adelgazar puede ayudarte a reducir el riesgo de padecer problemas de salud relacionados con el peso, como diabetes de tipo 2, presión arterial alta, apnea del sueño y enfermedades cardíacas, también puede presentar riesgos y complicaciones graves. (Vincentt 2015). Es posible que, a fin de someterte a la cirugía para bajar de peso, el paciente deba cumplir con ciertas pautas médicas, lo que indica que es probable que deba someterte a un extenso proceso de análisis para la detección de enfermedades con el objeto de determinar si es apto, o no para este tipo de procedimiento quirúrgico.

Por consiguiente, al hacer la evaluación para la cirugía de baipás gástrico, el equipo de atención médica tiene en cuenta lo siguiente:

- Cómo es la nutrición del paciente y sus antecedentes de peso. El equipo revisa las tendencias de peso, cómo han sido los intentos de seguir una dieta, sus hábitos alimentarios, su rutina de ejercicio, su nivel de estrés, sus restricciones temporales, su motivación y otros factores.

- Qué enfermedades padece. Algunos problemas de salud aumentan los riesgos asociados con someterse a una cirugía o pueden empeoran con esta, como los coágulos sanguíneos, la enfermedad hepática, los problemas cardíacos, los cálculos renales y las deficiencias nutricionales. El equipo evalúa qué medicamentos toma el paciente, cuánto alcohol ingiere, si posee el hábito del cigarrillo. De igual manera, le harán pruebas de apnea del sueño, además de un examen físico completo y un análisis de laboratorio. Los resultados de estos 


\section{Tratamiento y cuidados en cirugía de Bypass Gástrico}

Vol. 3, núm. 4., (2019)

María Fernanda Guiracocha Orellana; Nelson Ricardo Chacha Suscal; Gabriela Cecilia Arroyo Calvopiña; Silvia Jimena Zambrano Salvatierra; Mónica Gabriela Pozo Cárdenas; Javier Efraín Olivo Coello

análisis y exámenes podrían ayudar a determinar si el paciente es apto o no para la cirugía para adelgazar.

- Cómo está el estado psicológico del paciente. Algunos trastornos de salud mental podrían contribuir a provocar la obesidad o hacer que te resulte más difícil mantener los beneficios de la cirugía de baipás gástrico. Estos pueden comprender trastorno alimentario compulsivo, abuso de sustancias, trastornos de ansiedad, depresión mayor, esquizofrenia, trastorno bipolar grave y problemas relacionados con abuso sexual en la infancia.

Si bien es posible que estos trastornos no le impidan a la persona someterte a una cirugía de baipás gástrico, los médicos podrían posponer la cirugía para asegurarse de que cualquier afección o fuente importante de estrés esté tratada y controlada adecuadamente.

- Cómo está la motivación. El equipo también evaluará tu voluntad y tu capacidad para cumplir las recomendaciones que te haga el equipo de atención médica, así como para implementar los cambios recetados en tu dieta y en tu rutina de ejercicios.

- Qué edad tiene el paciente. No hay un límite de edad específico para someterse a una cirugía de baipás gástrico, pero, hasta hace poco, el procedimiento se consideraba riesgoso para adolescentes y para adultos mayores. Estudios más recientes determinaron que la cirugía de baipás gástrico puede ser segura y eficaz para adultos de 60 años o más. En la actualidad, este procedimiento también se considera una opción para algunos adolescentes con un índice de masa corporal de 35 o más y problemas de salud graves asociados a la obesidad.

\section{Riesgos Asociados al Bypass Gástrico}

Los riesgos asociados al bypass gástrico no son despreciables y puede producirse incluso el fallecimiento del paciente en aproximadamente el 0,35\% de los casos. Pero, según los expertos, los beneficios que el paciente obtiene para su salud superan claramente el riesgo, ya que con este 


\section{Tratamiento y cuidados en cirugía de Bypass Gástrico}

Vol. 3, núm. 4., (2019)

María Fernanda Guiracocha Orellana; Nelson Ricardo Chacha Suscal; Gabriela Cecilia Arroyo

Calvopiña; Silvia Jimena Zambrano Salvatierra; Mónica Gabriela Pozo Cárdenas; Javier Efraín Olivo Coello

tratamiento se reduce de forma más que significativa su riesgo de morir por un accidente cardiovascular, ya que la pérdida de peso que se obtiene permite reducir y controlar los factores de riesgo asociados a la obesidad mórbida.

\section{Imagen $N^{\circ}$ 2. Preparación médica para cirugía de Bypass Gástrico}

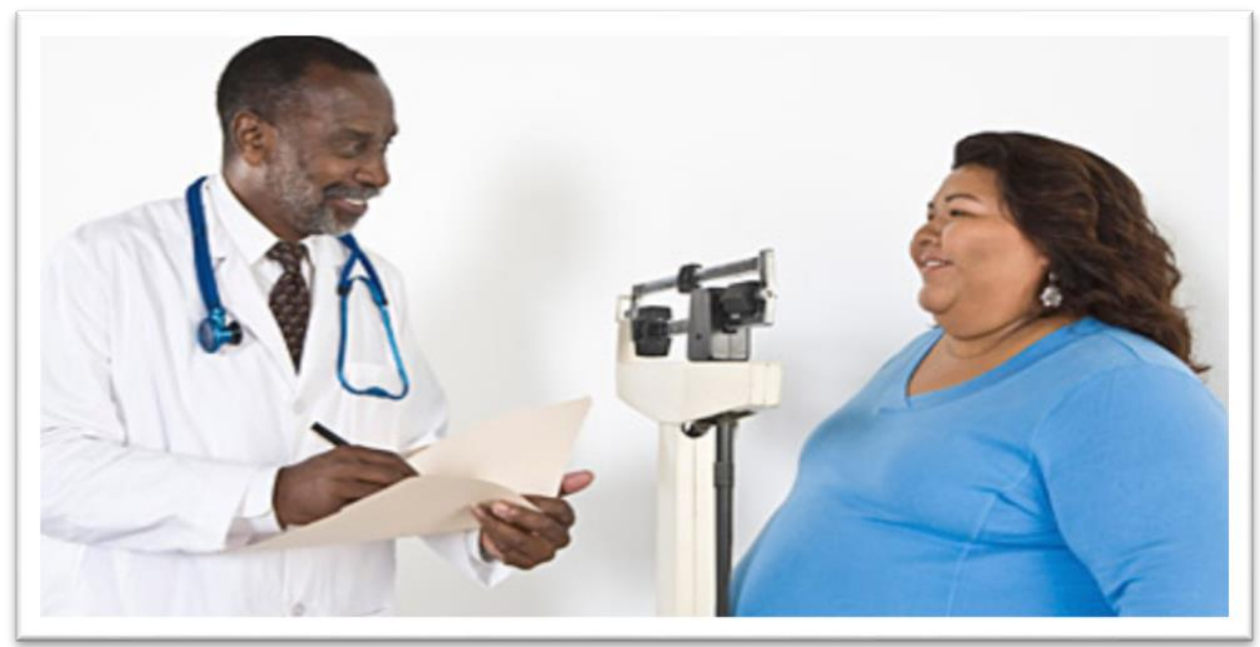

Fuente: (Vincent 2015)

Para ello es fundamental que estos pacientes cumplan con las garantías suficientes de que seguirán el tratamiento posterior a la cirugía a rajatabla, lo que implica una labor de reeducación en sus hábitos de vida (alimentación y ejercicio físico), que deberá mantener de forma permanente más allá de uno o dos años que se tarda en perder el exceso de peso. Ésta es la razón de que previamente tengan que superar una serie de pruebas médicas y de capacitación psicológica para someterse a este tipo de tratamiento, ya que de lo contrario se invertiría la relación beneficio-riesgo.

\section{Cuidado de la herida}

- La persona operada, podrá cambiar el apósito todos los días si su médico tratante así lo indica. Asegúrese de cambiar su apósito si se ensucia o se moja. Igualmente, deberá tener presente que puede tener hematomas alrededor de la herida, lo cual es normal y 


\section{Tratamiento y cuidados en cirugía de Bypass Gástrico}

Vol. 3, núm. 4., (2019)

María Fernanda Guiracocha Orellana; Nelson Ricardo Chacha Suscal; Gabriela Cecilia Arroyo Calvopiña; Silvia Jimena Zambrano Salvatierra; Mónica Gabriela Pozo Cárdenas; Javier Efraín Olivo Coello

desaparecerán espontáneamente, es decir, la piel alrededor de la incisión puede estar un poco roja, lo cual también es normal.

- La persona operada no deberá usar ropa apretada que haga fricción contra la incisión a medida que está sana.

- Mantenga el apósito (vendaje) sobre la herida limpio y seco. Si hay suturas o grapas, se las retirarán de 7 a 10 días después de la cirugía. Algunas suturas se pueden disolver por sí solas. Su proveedor de atención médica le dirá si usted las tiene.

- A menos que le digan lo contrario, el paciente no podrá ducharse hasta después de la cita de control con médico tratante. Cuando pueda bañarse, deje que el agua corra sobre la incisión, pero no la frote ni permita que el chorro la golpee.

- El paciente operado no deberá sumergirse en una bañera, piscina o jacuzzi hasta que su médico tratante le diga que puede hacerlo.

- El paciente operado deberá presionarse con una almohada sobre la incisión cuando necesite toser o estornudar.

\section{Medicamentos.}

Luego de la cirugía, es posible que el paciente con bypass gástrico necesite tomar algunos medicamentos cuando regrese a casa, generalmente las prescripciones médicas se basan en la dieta y el tipo de alimentos a consumir.

- Posiblemente necesite aplicarse inyecciones subcutáneas de un medicamento anticoagulante durante dos o más semanas. Su proveedor de atención médica le mostrará cómo hacerlo.

- Tal vez necesite tomar un medicamento para prevenir cálculos biliares.

- Necesitará tomar ciertas vitaminas que su cuerpo posiblemente no absorba bien de los alimentos. Dos de estas vitaminas son la vitamina B12 y la vitamina D.

- $\quad$ Es probable que también necesite tomar suplementos de calcio y hierro. 


\section{Tratamiento y cuidados en cirugía de Bypass Gástrico}

Vol. 3, núm. 4., (2019)

María Fernanda Guiracocha Orellana; Nelson Ricardo Chacha Suscal; Gabriela Cecilia Arroyo

Calvopiña; Silvia Jimena Zambrano Salvatierra; Mónica Gabriela Pozo Cárdenas; Javier Efraín Olivo Coello

El ácido acetilsalicílico (aspirin), el ibuprofeno (Advil, Motrin) y algunos otros fármacos pueden dañar el revestimiento del estómago o incluso ocasionar úlceras, por ello, es necesario que el paciente hable con su proveedor antes de tomar los medicamentos indicados.

\section{Beneficios del Bypass Gástrico}

- Aumento de la esperanza de vida: las personas con obesidad mórbida la tienen reducida considerablemente y no sólo por el importante riesgo de morbimortalidad cardiovascular que presentan, sino también por otras enfermedades para las que la obesidad es un factor importante de riesgo, como el cáncer.

- Calidad de vida: recuperan su capacidad para realizar las actividades cotidianas, desde las más simples, como vestirse, a otras que implican el desarrollo de una mayor y más satisfactoria actividad social.

- Un aumento significativo de su autoestima, tanto por la satisfacción de haber logrado eliminar el exceso de peso y mantenerse así, como por el hecho de poder disfrutar de la vida de una manera más saludable y satisfactoria.

\section{Control.}

Para ayudarlo a recuperarse de la cirugía y manejar todos los cambios en su estilo de vida, el paciente operado deberá consultar a su cirujano. Por ello, cuando salga del hospital, probablemente tendrá una cita de control programada con su cirujano al cabo de unas pocas semanas. (Cardozzo, 2017). Asimismo, el paciente, posterior a la cirugía debe llevar un control médico, el cual será pautado por el médico tratante, quien, le asignará asistencia médica de un nutricionista psicólogo y laboratorista de la siguiente manera:

- Un nutricionista o un dietista que le enseñarán cómo comer correctamente con el estómago más pequeño. También aprenderá acerca de qué alimentos y bebidas debe consumir después de la cirugía. 


\section{Tratamiento y cuidados en cirugía de Bypass Gástrico}

Vol. 3, núm. 4., (2019)

María Fernanda Guiracocha Orellana; Nelson Ricardo Chacha Suscal; Gabriela Cecilia Arroyo Calvopiña; Silvia Jimena Zambrano Salvatierra; Mónica Gabriela Pozo Cárdenas; Javier Efraín

Olivo Coello

- Un psicólogo, que puede ayudarlo a cumplir con las pautas de ejercicio y alimentación y hacerle frente a los sentimientos o preocupaciones que pueda tener después de la cirugía.

- Usted necesitará hacerse exámenes de sangre por el resto de su vida para constatar que su cuerpo esté obteniendo una cantidad suficiente de las vitaminas y los minerales importantes después de la cirugía.

La persona con bypass gástrico deberá contactar a un profesional médico su llegase a presentar las siguientes características:

- Tiene más enrojecimiento, dolor, calor al tacto, hinchazón o sangrado alrededor de la incisión.

- La herida es más grande o profunda o se ve oscura o reseca.

- La supuración proveniente de la incisión o de su alrededor no disminuye después de 3 a 5 días o aumenta.

- La supuración se torna espesa, de color marrón o amarillo o tiene mal olor (pus).

- Su temperatura está por encima de $100^{\circ} \mathrm{F}\left(37.7^{\circ} \mathrm{C}\right)$ durante más de 4 horas.

- Tiene dolor que no se alivia con un analgésico.

- Tiene problemas para respirar.

- Tiene una tos que no desaparece.

- No puede beber ni comer.

- La piel o la esclerótica de los ojos se torna de color amarillo.

- Las heces son sueltas o tiene diarrea.

- Está vomitando después de comer. 


\section{Tratamiento y cuidados en cirugía de Bypass Gástrico}

Vol. 3, núm. 4., (2019)

María Fernanda Guiracocha Orellana; Nelson Ricardo Chacha Suscal; Gabriela Cecilia Arroyo

Calvopiña; Silvia Jimena Zambrano Salvatierra; Mónica Gabriela Pozo Cárdenas; Javier Efraín Olivo Coello

\section{Riesgos.}

Los mayores riesgos de la dieta de bypass gástrico provienen de no seguir la dieta de forma adecuada. Si el paciente come demasiado o rompe la dieta (come alimentos que no debería), podría tener complicaciones. Algunas de ellas son:

- Síndrome de evacuación gástrica rápida. Si entra demasiada comida rápidamente en tu intestino delgado, es probable que experimentes náuseas, vómitos, mareos, sudoración y diarrea. Comer demasiado o demasiado rápido, comer alimentos ricos en grasa o azúcar y no masticar de forma adecuada los alimentos puede causar náuseas o vómitos después de las comidas.

- Deshidratación. Como se supone que no debes tomar líquidos con las comidas, algunas personas se deshidratan. Es por eso que debes beber 64 onzas (1.9 litros) de agua y otros líquidos en el día.

- Estreñimiento. La falta de actividad física y de fibra o líquidos en la dieta puede causar estreñimiento.

- Obstrucción de la abertura de la bolsa del estómago. La comida puede alojarse en la abertura de la bolsa del estómago, incluso si sigues cuidadosamente la dieta. Los signos y síntomas de una obstrucción de la abertura del estómago incluyen náuseas, vómitos y dolor abdominal continuos. Llama a tu médico si tienes estos síntomas durante más de dos días.

- Aumento de peso o incapacidad para perder peso. Si continúas aumentando de peso o no pierdes peso con la dieta de bypass gástrico, habla con tu médico o dietista.

\section{Peligros del Bypass Gástrico}

- No adelgazar a pesar del plan de adelgazamiento que sigue a la intervención (un 10\% de los casos). 


\section{Tratamiento y cuidados en cirugía de Bypass Gástrico}

Vol. 3, núm. 4., (2019)

María Fernanda Guiracocha Orellana; Nelson Ricardo Chacha Suscal; Gabriela Cecilia Arroyo Calvopiña; Silvia Jimena Zambrano Salvatierra; Mónica Gabriela Pozo Cárdenas; Javier Efraín Olivo Coello

- Complicaciones de la cirugía y el postoperatorio (un 15\%): hemorragias, infecciones, reacciones adversas a la anestesia, trombo embolismo, problemas respiratorios, etc.

- Complicaciones serias a largo plazo (2-3\%) que pueden incluso poner en riesgo su vida: obstrucción intestinal, síndrome de Dumping (diarreas, náuseas y vómitos), hernias, piedras en la vesícula, hipoglucemia, desnutrición etc.

- Fallecimiento del paciente (las estadísticas refieren la muerte de uno de cada 300 pacientes intervenidos).

\section{Objetivo.}

La dieta de bypass gástrico está diseñada para lo siguiente:

- Permitir que el estómago del paciente sane sin que la comida que consumes lo estire

- Está dirigida a que el paciente se acostumbre a comer las cantidades más pequeñas de alimentos que tu estómago más pequeño puede digerir de manera cómoda y segura

- La cirugía ayudará al paciente a perder peso y evitar aumentar de peso

- Evitar los efectos secundarios y las complicaciones de la cirugía.

\section{Detalles de la dieta.}

Las recomendaciones de alimentación después de la cirugía de bypass gástrico varían dependiendo de tu situación individual. Una dieta pos-bypass gástrico por lo general sigue un enfoque por etapas para ayudarte a volver a comer alimentos sólidos gradualmente. La rapidez con la que este plan de comidas pase de una etapa a la siguiente depende de la rapidez con la que el cuerpo del paciente se recupere y se adapte al cambio en los patrones de alimentación. Por lo general, estas personas pueden comenzar a comer alimentos de forma normal alrededor de tres meses después de la cirugía. (Cardozzo, 2017). De ahí, que cada etapa de la dieta pos-bypass gástrico, presta atención a lo siguiente: 


\section{Tratamiento y cuidados en cirugía de Bypass Gástrico}

Vol. 3, núm. 4., (2019)

María Fernanda Guiracocha Orellana; Nelson Ricardo Chacha Suscal; Gabriela Cecilia Arroyo

Calvopiña; Silvia Jimena Zambrano Salvatierra; Mónica Gabriela Pozo Cárdenas; Javier Efraín Olivo Coello

- Toma 64 onzas de líquido al día para evitar la deshidratación.

- Bebe líquidos entre comidas, no con las comidas. Espera alrededor de 30 minutos después de las comidas para beber y evita beber 30 minutos antes de las comidas.

- Come y bebe lentamente para evitar el síndrome de evacuación gástrica rápida, que ocurre cuando los alimentos y líquidos entran rápido al intestino delgado y en cantidades mayores a las normales y provocan náuseas, vómitos, mareos, sudoración y diarrea.

- Come alimentos magros y ricos en proteínas a diario.

- Elige alimentos y bebidas que sean bajos en grasas y azúcar.

- Evita el alcohol.

- Limita la cafeína, que puede causar deshidratación.

- Toma suplementos de vitaminas y minerales a diario según las indicaciones de tu proveedor de atención médica.

- Una vez que hayas pasado la etapa de líquidos solamente, mastica bien los alimentos hasta obtener una consistencia de puré antes de tragarlos.

\section{Líquidos}

Durante el primer día después de la cirugía, solo se le permitirá al paciente beber líquidos claros. Una vez que peste esté bien con los líquidos claros, puede comenzar a tomar otros líquidos, tales como:

- Caldo

- Jugo sin azúcar

- Té o café descafeinado 


\section{Tratamiento y cuidados en cirugía de Bypass Gástrico}

Vol. 3, núm. 4., (2019)

María Fernanda Guiracocha Orellana; Nelson Ricardo Chacha Suscal; Gabriela Cecilia Arroyo Calvopiña; Silvia Jimena Zambrano Salvatierra; Mónica Gabriela Pozo Cárdenas; Javier Efraín

Olivo Coello

- $\quad$ Leche (descremada o con $1 \%$ de grasa)

- Gelatina o paletas de helado sin azúcar.

\section{Alimentos hechos puré.}

Después de aproximadamente una semana de tolerar líquidos, el paciente podrá comenzar a comer alimentos colados y hechos puré. Los alimentos deben tener la consistencia de una pasta suave o de un líquido espeso, sin trozos sólidos de alimento en la mezcla.

Asimismo, podrá comer de tres a seis comidas pequeñas al día. Cada comida debe consistir de 4 a 6 cucharadas de alimento. Coma despacio: cada comida debe durar aproximadamente 30 minutos.

De igual forma, la persona elegirá alimentos que formen un buen puré, tales como:

- Carne de vaca molida magra, pollo o pescado

- Requesón

- Huevos revueltos blandos

- Cereal cocido

- Frutas blandas y vegetales cocidos

- Sopas cremas coladas.

Mezcla alimentos sólidos con un líquido, como:

- Agua

- Leche descremada

- Jugo sin azúcar añadido

- Caldo. 


\section{Tratamiento y cuidados en cirugía de Bypass Gástrico}

Vol. 3, núm. 4., (2019)

María Fernanda Guiracocha Orellana; Nelson Ricardo Chacha Suscal; Gabriela Cecilia Arroyo

Calvopiña; Silvia Jimena Zambrano Salvatierra; Mónica Gabriela Pozo Cárdenas; Javier Efraín Olivo Coello

\section{Alimentos blandos.}

Después de unas pocas semanas de comer alimentos en puré, y con la aprobación del médico tratante el paciente podrá agregar alimentos blandos a tu dieta. Deben ser trozos pequeños, tiernos y fáciles de masticar.

Asimismo, podrá comer de tres a cinco porciones pequeñas al día. Cada comida debe consistir de un tercio a media taza de comida. Mastica cada bocado hasta que la comida tenga consistencia de puré antes de tragarla. Los alimentos blandos incluyen:

- Carne de vaca molida magra o pollo

- Pescado desmenuzado

- Huevos

- Requesón

- Cereal cocido o seco

- Arroz

- Frutas frescas blandas o enlatadas, sin semillas ni piel

- Vegetales cocidos, sin piel

\section{Alimentos sólidos.}

Después de alrededor de ocho semanas con la dieta pos-bypass gástrico, el paciente podrá volver gradualmente a comer alimentos más firmes. Comienza con tres comidas al día. Cada porción debe consistir de 1 a 1-1/2 tazas de comida. Es importante dejar de comer antes de sentirse completamente lleno. 


\section{Tratamiento y cuidados en cirugía de Bypass Gástrico}

Vol. 3, núm. 4., (2019)

María Fernanda Guiracocha Orellana; Nelson Ricardo Chacha Suscal; Gabriela Cecilia Arroyo Calvopiña; Silvia Jimena Zambrano Salvatierra; Mónica Gabriela Pozo Cárdenas; Javier Efraín Olivo Coello

Según la forma cómo el paciente tolere los alimentos sólidos, es posible que puedas variar el número de comidas y la cantidad de alimentos en cada comida. El paciente deberá hablar con su nutricionista y dietista sobre lo que es mejor para él.

La persona operada deberá probar alimentos nuevos de a uno por vez. Ciertos alimentos pueden causar dolor, náuseas o vómitos después de la cirugía de bypass gástrico.

Los alimentos que pueden causar problemas en esta etapa incluyen:

- Panes

- Bebidas gaseosas

- Vegetales crudos

- Vegetales fibrosos cocidos, como apio, brócoli, maíz o repollo

- Carnes duras o carnes con cartílago

- Carnes rojas

- Comidas fritas

- Comidas muy condimentadas o picantes

- Frutos secos y semillas

- Palomitas de maíz.

Con el tiempo, es posible que la persona logre probar algunos de estos alimentos de nuevo, todo esto, deberá estar apoyado en orientaciones del médico tratante.

Una nueva dieta saludable 


\section{Tratamiento y cuidados en cirugía de Bypass Gástrico}

Vol. 3, núm. 4., (2019)

María Fernanda Guiracocha Orellana; Nelson Ricardo Chacha Suscal; Gabriela Cecilia Arroyo

Calvopiña; Silvia Jimena Zambrano Salvatierra; Mónica Gabriela Pozo Cárdenas; Javier Efraín Olivo Coello

La cirugía de bypass gástrico reduce el tamaño de tu estómago y cambia la manera en que los alimentos ingresan a los intestinos de las personas. Después de la cirugía, es importante que el paciente se alimente de forma adecuada, mientras se mantiene a tono con los objetivos de pérdida de peso. Es probable que el médico le recomiende que:

- Come y bebe despacio. Para evitar el síndrome de evacuación gástrica rápida, tómate al menos 30 minutos para comer y de 30 a 60 minutos para beber 1 taza de líquido. Espera 30 minutos antes o después de cada comida para tomar líquidos.

- Come cantidades pequeñas. Come varias comidas pequeñas al día. Puedes comenzar con seis comidas pequeñas al día, luego pasar a cuatro comidas y, por último, cuando sigas una dieta regular, tres comidas al día. Cada comida debe incluir entre media taza y una taza de comida.

- Bebe líquidos entre las comidas. Para evitar la deshidratación, necesitarás beber por lo menos 8 tazas (1.9 litros) de líquidos al día. Pero beber demasiado líquido a la hora de comer o cerca de las comidas puede hacer que te sientas demasiado lleno y evitar que comas suficientes alimentos ricos en nutrientes.

- Mastica los alimentos por completo. La nueva abertura que va del estómago al intestino delgado es muy estrecha y bloquearse con trozos grandes de comida. Las obstrucciones impiden que los alimentos salgan del estómago y pueden causar vómitos, náuseas y dolor abdominal. Toma pequeños bocados de comida y mastícalos hasta obtener una consistencia de puré antes de tragarlos.

- Prioriza los alimentos ricos en proteínas. Come estos alimentos antes de comer otros alimentos en tu comida.

- Evita los alimentos con alto contenido de grasa y azúcar. Estos alimentos viajan rápidamente a través de tu sistema digestivo y causan el síndrome de evacuación gástrica rápida. 


\section{Tratamiento y cuidados en cirugía de Bypass Gástrico}

Vol. 3, núm. 4., (2019)

María Fernanda Guiracocha Orellana; Nelson Ricardo Chacha Suscal; Gabriela Cecilia Arroyo Calvopiña; Silvia Jimena Zambrano Salvatierra; Mónica Gabriela Pozo Cárdenas; Javier Efraín Olivo Coello

- Toma los suplementos de vitaminas y minerales recomendados. Después de la cirugía, tu cuerpo no será capaz de absorber suficientes nutrientes de los alimentos. Es probable que necesites tomar un suplemento multivitamínico todos los días por el resto de tu vida.

\section{Conclusiones.}

La cirugía de bypass gástrico representa una técnica quirúrgica, realizada de manera general para personas obesas, con el fin de ayudarle a los mismos a adoptar gradualmente una manera saludable de comer, la cual les permita lograr sus metas para bajar de peso. Los objetivos del cuidado nutricional luego de este tipo de cirugías restrictivas son básicamente dos: por un lado, se necesita un adecuado aporte energético y nutricional para la recuperación post quirúrgica y la preservación de masa magra durante la pérdida importante de peso; por otro lado, los alimentos ingeridos luego de la cirugía deben minimizar el reflujo y la saciedad precoz, maximizando la pérdida de peso

En términos generales, el cuidado post operatorio se reduce de manera general a la dieta que debe llevar el paciente, llamadas post cirugías restrictivas o bypass gástrico, la cual se desarrolla en varias fases, con progresión de líquidos a sólidos. Durante un período de 6-8 semanas el paciente progresa a través de 4 fases: dieta líquida (líquidos claros y luego líquidos completos), dieta puré, dieta semisólida y, por último, dieta sólida, focalizándose siempre en las necesidades de líquidos y proteínas. La primera fase tras la cirugía consiste en la administración de líquidos claros, durante 2-3 días, para seguir con una dieta líquida completa. Algunas de las alternativas dietéticas para esta fase pueden incluir yogur líquido, preferentemente con proteínas añadidas.

A través de estos cuidados, el paciente progresa en consistencia a una dieta triturada o blanda. La dieta triturada incluye alimentos muy blandos y ricos en proteínas, como huevo, quesos bajos en calorías y carnes magras de pollo, vacuno, cerdo o pescado (las carnes rojas son peor toleradas). Es importante incorporar proteínas de alto valor biológico en cada comida, como claras de huevo, carnes magras, quesos o leche. El objetivo nutricional es que el paciente consuma una dieta balanceada que incluya porciones adecuadas de todos los grupos de alimentos y limite los azúcares agregados, dulces concentrados, frituras, gaseosas, cafeína y alcohol; ara el logro de estos 


\section{Tratamiento y cuidados en cirugía de Bypass Gástrico}

Vol. 3, núm. 4., (2019)

María Fernanda Guiracocha Orellana; Nelson Ricardo Chacha Suscal; Gabriela Cecilia Arroyo

Calvopiña; Silvia Jimena Zambrano Salvatierra; Mónica Gabriela Pozo Cárdenas; Javier Efraín Olivo Coello

objetivos, se entrena a los pacientes para que consuman las necesidades mínimas diarias de hidratos de carbono (100-130 g/d) y grasas (20 g/d), en base a la RDA para adultos.

\section{Bibliografía.}

Arias, F. (2010). Paradigmas de la Investigación Científica. España: Editorial: Luces.

Cardozzo, C. (2017). Beneficios que brinda el Bypass Gástrico a pacientes obesos. Revista Vida y Salud de la Universidad de la Habana en Cuba., 43.

Dávila, N. (2012). Paradigmas de la Investigación Científica. Pereire, Colombia: Editorial: Las Brisas.

Herrsent, G. (2016). Bypass Gástrico en pacientes depresivos. Revista de Salud Integral de la Universidad Autónoma de México., 23.

Vianco, S. (2016). Tratamiento y cuidados del Bypass Gástrico. Revista de Publicaciones Médicas de España., 14.

Vincent, R. (2015). écnicas Quirúrgicas en Cirugías Venosas, Recanalización de la Vena Sefana. Revista Española de Cirugía , 54.

$$
\text { (9) }(\mathcal{Q} \Theta
$$

RECONOCIMIENTO-NOCOMERCIAL-COMPARTIRIGUAL

CC BY-NC-SA

ESTA LICENCIA PERMITE A OTROS ENTREMEZCLAR, AJUSTAR Y CONSTRUIR A PARTIR DE SU OBRA CON FINES NO

COMERCIALES, SIEMPRE Y CUANDO LE RECONOZCAN LA AUTORÍA Y SUS NUEVAS CREACIONES ESTÉN BAJO UNA LICENCIA CON LOS MISMOS TÉRMINOS. 